Bull. Austral. Math. Soc.

$26 \mathrm{E} 25,54 \mathrm{C} 65$

VOL. 65 (2002) [407-422]

\title{
ON CONTINUITY AND SELECTIONS OF MULTIFUNCTIONS
}

\author{
PANDELIS DODOS
}

\begin{abstract}
The notions of a Baire-1 and a weak Baire-1 multifunction are defined and a striking analogy between Baire-1 multifunctions and classical Baire-1 functions is established. A selection theorem is presented which asserts that if $X$ is a metrisable space, $Y$ a Polish space and $F: X \rightarrow 2^{Y} \backslash\{\emptyset\}$ a closed-valued, weak Baire-1 multifunction, then $F$ admits a Baire-1 selection. Using the machinery developed we prove that if $X$ is a Banach space with separable dual, then every weak* usco, defined on a completely metrisable space $Z$, which values are weakly* compact subsets of the dual, is norm lower semicontinuous on a dense $G_{\delta}$ set.
\end{abstract}

\section{INTRODUCTION}

The purposes of this paper are two:

(i) to indicate that certain facts about generic continuity of multifunctions can be derived from the theory of Baire-1 functions;

(ii) to demonstrate that a large class of multifunctions (which includes the upper semicontinuous and lower semicontinuous ones) admit a Baire-1 selection. It is organised as follows.

In Section (2) some necessary mathematical background is presented. We also give a number of technical lemmas, which concern the behaviour of the weak and the strong inverse image of a multifunction, and are crucial for our future considerations.

In Section (3) the notions of Baire-1 and weak Baire-1 multifunction are defined in the context of metrisable spaces, via complexity of weak and strong inverse images of open sets (both definitions are equivalent to the classical definition of Baire-1 functions for single-valued multifunctions). We show that every upper semicontinuous multifunction is a Baire-1 multifunction and every lower semicontinuous is a weak Baire-1 multifunction. When $Y$ is a separable metrisable space, we prove that a compact-valued multifunction is a Baire-1 multifunction if and only if it is a Baire-1 function, viewed as a single-valued function which takes values in the Vietoris hyperspace topology of compact subsets of $Y$. This is a key result which permits us to derive standard facts about continuity of multifunctions almost directly.

Received 25th September, 2001

Copyright Clearance Centre, Inc. Serial-fee code: 0004-9727/02 \$A2.00+0.00. 
In Section 4 we turn our attention to the possibility of finding "good" selections of Baire-1 multifunctions. The main theorem of this section states that if $X$ is a metrisable space, $Y$ a Polish space and $F$ a weak Baire-1 closed-valued multifunction, then $F$ admits a Baire-1 selection. It is modelled after the Kuratowski-Ryll Nardzewski selection theorem and it is based upon the generalised reduction property of $F_{\sigma}$ sets. We should point out that there exist a large number of papers in the literature dealing with approximate or generically continuous selections of multifunctions. We do not intend to survey all of them. We refer to [2] or to [5] for more information. However we should notice that our selection theorem is general enough for three reasons:

(i) it is valid for a large class of multifunctions (which essentially includes the upper and lower semicontinuous ones);

(ii) it unifies the approach;

(iii) it does not require linear structure on the range space (so it can be applied at the lack of convexity).

In Section 5 we consider weak* uscos (weak* cuscos) defined on a completely metrisable space $Z$ and taking values in the weakly* compact (convex) subsets of the dual of a Banach space. We prove that if $X$ is a Banach space with a separable dual $X^{*}$ and $F: Z \rightarrow P_{w k}\left(X_{w^{*}}^{*}\right)$ a weak ${ }^{*}$ usco, then $F$ is norm lower semicontinuous on a dense $G_{\delta}$ set. If in addition $F$ has strongly compact values, then we show that $F$ is norm continuous on a dense $G_{\delta}$ set. Our proofs are essentially based upon the technics developed in the previous two sections, and especially on the results of Section 3 .

In the last section we show that our selection theorem is sharp in the following sense: it is essentially equivalent to the generalised reduction property of $F_{\sigma}$ sets. Specifically we prove the following: let $X$ be a metrisable space. If for any Polish space $Y$, any weak Baire-1 multifunction $F: X \rightarrow P_{f}(Y)$ admits a Baire-1 selection, then the class of $F_{\sigma}$ subsets of $X$ has the generalised reduction property.

\section{Mathematical Preliminaries}

For any topological space $X, P_{f}(X)$ and $P_{k}(X)$ denote the collections of all nonempty closed and nonempty compact subsets of $X$ respectively. As usual, by $N(x)$ we denote the filter of neighbourhoods of $x \in X$.

Let us recall some definitions from multivalued analysis. Let $X$ and $Y$ be Hausdorf topological spaces. For any multifunction (set-valued map) $F: X \rightarrow 2^{Y} \backslash\{\emptyset\}$ and any set $A \subseteq Y$ one defines the weak inverse image of $A$ under $F$ by $F^{-}(A)=\{x \in X$ : $F(x) \bigcap A \neq \emptyset\}$. Similarly the strong inverse image of $A$ under $F$ is defined by $F^{+}(A)=$ $\{x \in X: F(x) \subseteq A\}$. It is easy to see that for any $A \subseteq Y$ it holds that $F^{-}(A)=$ $\left(F^{+}\left(A^{c}\right)\right)^{c}$. In addition if $\left\{A_{i}\right\}_{i \in I} \subseteq Y$ we have that:

$$
F^{-}\left(\bigcup_{i \in I} A_{i}\right)=\bigcup_{i \in I} F^{-}\left(A_{i}\right), F^{-}\left(\bigcap_{i \in I} A_{i}\right) \subseteq \bigcap_{i \in I} F^{-}\left(A_{i}\right)
$$


(ii) $F^{+}\left(\bigcup_{i \in I} A_{i}\right) \supseteq \bigcup_{i \in I} F^{+}\left(A_{i}\right), F^{+}\left(\bigcap_{i \in I} A_{i}\right)=\bigcap_{i \in I} F^{+}\left(A_{i}\right)$.

The following definitions are standard.

DEFINITION 1: Let $F: X \rightarrow 2^{Y} \backslash\{\emptyset\}$ be a multifunction.

(i) $F$ is said to be upper semicontinuous if and only if $F^{+}(U)$ is open in $X$, for every $U \subseteq Y$ open.

(ii) $F$ is said to be lower semicontinuous if and only if $F^{-}(U)$ is open in $X$, for every $U \subseteq Y$ open.

(iii) $F$ is said to be continuous if and only if both $F^{+}(U)$ and $F^{-}(U)$ are open in $X$, for every $U \subseteq Y$ open.

We can have local concepts of the above notions. So $F$ is said to be upper semicontinuous at $x_{0} \in X$ if and only if given $U \subseteq Y$ open such that $F\left(x_{0}\right) \subseteq U$ we can find $V \in N\left(x_{0}\right)$ such that $F(V) \subseteq U$ (that is, $F^{+}(U) \supseteq V$ ). Similarly one defines the local notions of lower semicontinuity and continuity. For more information about continuity concepts of multifunctions, one can consult [5].

We widely use the definitions and notations from descriptive set theory. So for a metrisable space $X$ by $\Sigma_{1}^{0}(X)$ we denote the open subsets of $X$, by $\Pi_{1}^{0}(X)$ the closed, by $\Sigma_{2}^{0}(X)$ the $F_{\sigma}$, by $\Pi_{2}^{0}(X)$ the $G_{\delta}$, et cetera. For more information we refer to [6].

Recall that if $X$ and $Y$ are metrisable spaces, then a function $f: X \rightarrow Y$ is said to be a Baire-1 function if and only if $f^{-1}(U) \in \boldsymbol{\Sigma}_{2}^{0}(X)$ for every $U \subseteq Y$ open. Baire-1 functions have been studied extensively and have found remarkable applications (see for instance [11]). Of course one can go beyond the class of Baire-1 functions and define higher classes of Baire functions. So recursively, for $1<\xi<\omega_{1}$, we define a function $f: X \rightarrow Y$ to be a Baire- $\xi$ function if and only if $f$ is the pointwise limit of a sequence $\left\{f_{n}\right\}_{n \geqslant 1}$ of Baire- $\xi_{n}$ functions, where $\xi_{n}<\xi$.

Recall that if $\Gamma$ is a given class of sets in a metrisable space $X$, then we say that a function $f: X \rightarrow Y$ is $\Gamma$-measurable if $f^{-1}(U) \in \Gamma$ for any $U \subseteq Y$ open. So the $\Sigma_{1}^{0}(X)$-measurable functions are the continuous ones while the $\Sigma_{2}^{0}(X)$-measurable are the Baire-1 functions.

When $\Gamma$ is a given class of sets, we say that $\Gamma$ has the generalised reduction property . if for any sequence $\left\{A_{n}\right\}_{n \geqslant 1} \subseteq \Gamma$ there exists a sequence $\left\{B_{n}\right\}_{n \geqslant 1} \subseteq \Gamma$ such that $B_{n} \subseteq A_{n}$, $B_{n} \cap B_{m}=\emptyset$ for $n \neq m$ and $\bigcup_{n \geqslant 1} A_{n}=\bigcup_{n \geqslant 1} B_{n}$. In a metrisable space $X$, for any $\xi>1$, the class $\Sigma_{\xi}^{0}(X)$ has the generalised reduction property (see [6, p. 172]). This also holds if $\xi=1$ for zero-dimensional spaces.

We close this section by presenting some rather technical lemmas which will be useful for our future considerations. We show that the strong (respectively the weak) inverse image of a multifunction $F: X \rightarrow 2^{Y} \backslash\{\emptyset\}$, behaves well at least with respect to unions (respectively intersections) of monotone families of sets. Our arguments are based on compactness, so we restrict ourselves to $P_{k}(Y)$-valued multifunctions. 
Lemma 2. Let $X, Y$ be Hausdorff topological spaces and $F: X \rightarrow P_{k}(Y)$ a multifunction. Let $\left\{A_{n}\right\}_{n} \geqslant 1$ be an increasing sequence of open subsets of $Y$ (that is, $A_{n} \supseteq A_{m}$ if $\left.n \geqslant m\right)$. Then $F^{+}\left(\bigcup_{n \geqslant 1} A_{n}\right)=\bigcup_{n \geqslant 1} F^{+}\left(A_{n}\right)$.

ProOF: Let $\left\{A_{n}\right\}_{n \geqslant 1}$ be an increasing sequence of open subsets of $Y$. Clearly we have that $F^{+}\left(\bigcup_{n \geqslant 1} A_{n}\right) \supseteq \bigcup_{n \geqslant 1} F^{+}\left(A_{n}\right)$. So we only have to check the opposite inclusion. Let $x \in F^{+}\left(\bigcup_{n \geqslant 1} A_{n}\right)$, which implies that $F(x) \subseteq \bigcup_{n \geqslant 1} A_{n}$. The family $\left\{A_{n}\right\}_{n \geqslant 1}$ forms an open cover of $F(x)$. Since the latter is compact, there exists a finite subcover, say $\left\{A_{n}\right\}_{n=1}^{k}$. Hence we get $F(x) \subseteq \bigcup_{n=1}^{k} A_{n}$. By hypothesis $\left\{A_{n}\right\}_{n \geqslant 1}$ is an increasing sequence of sets, so there exists some $A_{i} \in\left\{A_{n}\right\}_{n=1}^{k}$ such that $A_{i} \supseteq A_{n}$ for every $n=1, \ldots, k$. Thus $F(x) \subseteq A_{i}$, which implies that $x \in F^{+}\left(A_{i}\right)$ and in particular that $x \in \bigcup_{n \geqslant 1} F^{+}\left(A_{n}\right)$. So we conclude that $F^{+}\left(\bigcup_{n \geqslant 1} A_{n}\right)=\bigcup_{n \geqslant 1} F^{+}\left(A_{n}\right)$, as desired.

The following lemma is a variant of Lemma 2.

Lemma 3. Let $X, Y$ be Hausdorf topological spaces and $F: X \rightarrow P_{k}(Y)$ a multifunction. Let $\left\{A_{n}\right\}_{n \geqslant 1}$ be a sequence of nonempty open subsets of $Y$ such that $A_{n} \supseteq \overline{A_{n+1}}$ for every $n \geqslant 1$. Then $F^{-}\left(\bigcap_{n \geqslant 1} A_{n}\right)=\bigcap_{n \geqslant 1} F^{-}\left(A_{n}\right)$.

Proof: Let $\left\{A_{n}\right\}_{n \geqslant 1}$ be a sequence of nonempty open subsets of $Y$ as described above. It is clear that $F^{-}\left(\bigcap_{n \geqslant 1} A_{n}\right) \subseteq \bigcap_{n \geqslant 1} F^{-}\left(A_{n}\right)$. Assume that the inclusion was strict. Then there exists some $x \in \bigcap_{n \geqslant 1} F^{-}\left(A_{n}\right)$ such that $x \notin F^{-}\left(\bigcap_{n \geqslant 1} A_{n}\right)$. This implies that $F(x) \cap A_{n} \neq \emptyset$ for every $n \geqslant 1$ and $F(x) \cap\left(\bigcap_{n \geqslant 1} A_{n}\right)=\emptyset$. For every $n \geqslant 1$ define $C_{n}=F(x) \cap \overline{A_{n+1}} \neq \emptyset$. Note that the sequence $\left\{C_{n}\right\}_{n \geqslant 1}$ is a (strictly) decreasing sequence of closed sets in $F(x)$, the latter equipped with the relative topology. So it has the finite intersection property. Since $F(x)$ is compact we conclude that $\bigcap_{n \geqslant 1} C_{n} \neq \emptyset$. But observe that $F(x) \cap\left(\bigcap_{n \geqslant 1} A_{n}\right)=F(x) \cap\left(\bigcap_{n \geqslant 1} C_{n}\right) \neq \emptyset$, which is clearly a contradiction. So we conclude that $F^{-}\left(\bigcap_{n \geqslant 1} A_{n}\right)=\bigcap_{n \geqslant 1} F^{-}\left(A_{n}\right)$.

REMARK 1. We can have dual versions of Lemma 2 and Lemma 3. So, for instance, from Lemma 2, we get that if $X, Y$ and $F$ are as above, then for any decreasing sequence $\left\{C_{n}\right\}_{n \geqslant 1}$ of (nonempty) closed subsets of $Y$ it holds that $F^{-}\left(\bigcap_{n \geqslant 1} C_{n}\right)=\bigcap_{n \geqslant 1} F^{-}\left(C_{n}\right)$.

The last lemma indicates in which sequence of sets Lemma 3 will be applied.

Lemma 4. Let $X$ be a metrisable space. If $C$ is nonempty closed subset of $X$, then there exists a decreasing sequence $\left\{U_{n}\right\}_{n \geqslant 1}$ of open subsets of $X$ such that $U_{n} \supseteq \overline{U_{n+1}}$ for every $n \geqslant 1$ and $C=\bigcap_{n \geqslant 1} U_{n}$.

Proof: Let $d$ be a compatible metric for $X$ and $C \in \Pi_{1}^{0}(X)$. For every $x \in X$ put 
$d(x, C)=\inf \{d(x, c): c \in C\}$. The function $x \rightarrow d(x, C)$ is continuous (see [6, p. 15]). Define $U_{n}=\{x \in X: d(x, C)<1 / n\}$ for every $n \geqslant 1$. Clearly $\left\{U_{n}\right\}_{n \geqslant 1}$ is the desired sequence of open subsets of $X$.

\section{Baire-1 Multifunctions}

Unless otherwise stated, through this section $X$ and $Y$ will always be metrisable spaces.

Definition 5: A multifunction $F: X \rightarrow 2^{Y} \backslash\{\theta\}$ is said to be a "Baire-1 multifunction" if and only if $F^{-}(U) \in \Sigma_{2}^{0}(X)$ and $F^{+}(U) \in \Sigma_{2}^{0}(X)$ for any $U \subseteq Y$ open. It is said to be a "weak Baire-1 multifunction" if and only if $F^{-}(U) \in \boldsymbol{\Sigma}_{2}^{0}(X)$ for any $U \subseteq Y$ open.

Obviously each Baire-1 multifunction is a weak Baire-1 multifunction. It is also clear that if $F$ is single-valued then both definitions are equivalent to the usual definition of Baire-1 functions.

EXAMPLE 1.

(i) Let $F: \mathbb{R} \rightarrow P_{k c}(\mathbb{R})$ defined by:

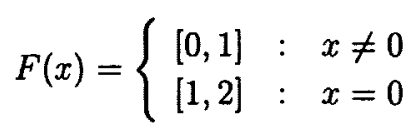

Then $F^{-}((3 / 2,3))=\{0\}$ and $F^{+}((1 / 2,3))=\{0\}$. So $F$ is neither upper nor lower semicontinuous. However it is easy to check that it is Baire-1.

(ii) Define $F: \mathbb{R} \rightarrow P_{k c}(\mathbb{R})$ by:

$$
F(x)=\left\{\begin{array}{lll}
{[0,1]} & : & x>0 \\
{[-2,-1]} & : & x \leqslant 0
\end{array}\right.
$$

Observe that $F$ is Baire-1 and that $F$ can not have a continuous selection.

(iii) Put $A=[0,1] \cap \mathbb{Q}$ and define $F:[0,1] \rightarrow P_{k c}(\mathbb{R})$ by:

$$
F(x)=\left\{\begin{array}{lll}
{[0,1]} & : & x \in A \\
0 & : & x \notin A
\end{array}\right.
$$

Observe that $F^{+}((-1,1))=[0,1] \backslash A$ which is $G_{\delta}$ but not $F_{\sigma}$. So $F$ is not Baire-1 multifunction. But it is weak Baire-1. Indeed, if $U \subseteq \mathbb{R}$ is open, then $F^{-}(U)=[0,1]$ if $U \cap\{0\} \neq \emptyset, F^{-}(U)=A$ if $U \cap[0,1] \neq \emptyset$ and $U \cap\{0\}=\emptyset$, and $F^{-}(U)=\emptyset$ otherwise. So $F^{-}(U)$ is $F_{\sigma}$ for every $U \subseteq \mathbb{R}$ open.

From the identity $F^{-}(A)=\left(F^{+}\left(A^{c}\right)\right)^{c}$ for any $A \subseteq Y$, we have the following. 
Proposition 6. A multifunction $F: X \rightarrow 2^{Y} \backslash\{\emptyset\}$ is a Baire-1 multifunction if and only if $F^{-}(C) \in \Pi_{2}^{0}(X)$ and $F^{+}(C) \in \Pi_{2}^{0}(X)$ for any $C \subseteq Y$ closed. Respectively $F$ is a weak Baire-1 multifunction if and only if $F^{+}(C) \in \Pi_{2}^{0}(X)$ for any $C \subseteq Y$ closed.

Using Lemma 3 and Lemma 4, we shall show that every upper semicontinuous multifunction and every $P_{k}(Y)$-valued lower semicontinuous multifunction is a Baire-1 multifunction. In general lower semicontinuous multifunctions are weak Baire-1 multifunctions.

Proposition 7. We have the following.

(i) If $F: X \rightarrow 2^{Y} \backslash\{\emptyset\}$ is an upper semicontinuous multifunction, then $F$ is a Baire-1 multifunction.

(ii) If $F: X \rightarrow 2^{Y} \backslash\{\emptyset\}$ is a lower semicontinuous multifunction, then $F$ is a weak Baire-1 multifunction.

(iii) If $F: X \rightarrow P_{k}(Y)$ is a lower semicontinuous multifunction, then $F$ is a Baire-1 multifunction.

Proof: (i) Assume that $F$ is upper semicontinuous. Let $U \subseteq Y$ be open. Then we have that $F^{+}(U)$ is an open subset of $X$. Recall that in metrisable spaces open sets are $F_{\sigma}$. Thus $F^{+}(U) \in \Sigma_{2}^{0}(X)$. So we only need to prove that $F^{-}(U)$ is $F_{\sigma}$. Since $U$ is open subset of $Y$ and $Y$ is a metrisable space, we have that $U$ is $F_{\sigma}$. Thus $U=\bigcup_{n \geqslant 1} C_{n}$, where $C_{n} \subseteq Y$ are closed for any $n \geqslant 1$. Observe that:

$$
F^{-}(U)=F^{-}\left(\bigcup_{n \geqslant 1} C_{n}\right)=\bigcup_{n \geqslant 1} F^{-}\left(C_{n}\right) .
$$

Since $F$ is upper semicontinuous, $F^{-}(C)$ is closed for any $C \subseteq Y$ closed. So we conclude that $F^{-}(U) \in \boldsymbol{\Sigma}_{2}^{0}(X)$ as desired.

(ii) It follows immediately from the definition of lower semicontinuity and the fact that in metrisable spaces open sets are $F_{\sigma}$.

(iii) Now assume that $F$ is lower semicontinuous and $P_{k}(Y)$-valued. In light of Proposition 6, we need to prove that both $F^{-}(C)$ and $F^{+}(C)$ are $G_{\delta}$ subsets of $X$ for any $C \subseteq Y$ closed. From part (ii) it is clear that we only have to prove that $F^{-}(C) \in \Pi_{2}^{0}(X)$. Let $C \subseteq Y$ closed. Apply Lemma 4 and get a decreasing sequence $\left\{V_{n}\right\}_{n \geqslant 1}$ of open subsets of $Y$ such that $V_{n} \supseteq \overline{V_{n+1}}$ for every $n \geqslant 1$ and $C=\bigcap_{n \geqslant 1} V_{n}$. Invoking Lemma 3 we
have that:

$$
F^{-}(C)=F^{-}\left(\bigcap_{n \geqslant 1} V_{n}\right)=\bigcap_{n \geqslant 1} F^{-}\left(V_{n}\right)
$$

Since $F$ is lower semicontinuous, $F^{-}(U)$ is open for any $U \subseteq Y$ open. So we conclude that $F^{-}(C) \in \Pi_{2}^{0}(X)$ and this completes the proof.

Now assume that $Y$ is a separable metrisable space. Recall that $P_{k}(Y)$ equipped with the Vietoris hyperspace topology (still denoted by $\left(P_{k}(Y), \tau_{V}\right)$ ) is also separable metrisable and in particular second countable (see for instance [5, p. 15] or [6, p. 25]). The following proposition demonstrates that for the case of $P_{k}(Y)$-valued multifunctions, 
there is a strong relationship between Baire-1 multifunctions and Baire-1 functions from $X$ into $\left(P_{k}(Y), \tau_{V}\right)$.

PROPOS ITION 8. Let $Y$ be a separable metrisable space and $F: X \rightarrow P_{k}(Y)$. Then $F$ is a Baire-1 multifunction if and only if $F$ is a Baire-1 function, viewed as a single-valued function from $X$ into $\left(P_{k}(Y), \tau_{V}\right)$.

Proof: Let $F$ be a Baire-1 multifunction. We need to prove that $F^{-1}(W) \in \Sigma_{2}^{0}(X)$ for any open set $W$ of $\left(P_{k}(Y), \tau_{V}\right)$. Recall that basic open sets in $\left(P_{k}(Y), \tau_{V}\right)$ are of the form:

$$
B\left(U, V_{1}, \ldots, V_{k}\right)=\left\{A \in P_{k}(Y): A \subseteq U, A \cap V_{n} \neq \emptyset, n=1, \ldots, k\right\}
$$

for some $U, V_{1}, \ldots, V_{k} \subseteq Y$ open. We have that:

$$
\begin{aligned}
F^{-1}\left(B\left(U, V_{1}, \ldots, V_{k}\right)\right) & =\left\{x \in X: F(x) \subseteq U, F(x) \cap V_{n} \neq \emptyset, n=1, \ldots, k\right\} \\
& =F^{+}(U) \cap F^{-}\left(V_{1}\right) \cap \ldots \cap F^{-}\left(V_{k}\right) \in \Sigma_{2}^{0}(X) .
\end{aligned}
$$

Since $\left(P_{k}(Y), \tau_{V}\right)$ is second countable, any open set $W$ of $\left(P_{k}(Y), \tau_{V}\right)$ can be written as a countable union of basic open sets. So we conclude that $F^{-1}(W) \in \Sigma_{2}^{0}(X)$, which implies that $F$ is a Baire-1 function from $X$ into $\left(P_{k}(Y), \tau_{V}\right)$.

Conversely, assume that $F$ is a Baire-1 function from $X$ into $\left(P_{k}(Y), \tau_{V}\right)$. Let $U \subseteq Y$ open. The set $B(U, Y)=\left\{A \in P_{k}(Y): A \subseteq U, A \cap Y \neq \emptyset\right\}$ is clearly open in $\left(P_{k}(Y), \tau_{V}\right)$. We have that:

$$
\begin{aligned}
F^{-1}(B(U, Y)) \in \Sigma_{2}^{0}(X) & \Leftrightarrow\{x \in X: F(x) \subseteq U, F(x) \cap Y \neq \emptyset\} \in \Sigma_{2}^{0}(X) \\
& \Leftrightarrow F^{+}(U) \in \Sigma_{2}^{0}(X) .
\end{aligned}
$$

Similarly, by taking $B(Y, U)$, we conclude that $F^{-}(U) \in \Sigma_{2}^{0}(X)$. Thus $F$ is a Baire-1 multifunction.

Recall that if $X$ is Polish and $Y$ is separable metrisable, then a function $f: X \rightarrow Y$ is Baire-1 if and only if $\left.f\right|_{C}$ has a point of continuity for every nonempty closed set $C \subseteq X$ (see [6, pp. 193-194]). So from Proposition 8 we have that if $X$ is Polish then $F: X \rightarrow P_{k}(Y)$ is a Baire-1 multifunction if and only if $\left.F\right|_{C}$ has a point of continuity for every $C \subseteq X$ closed. It is also well-known that if $f: X \rightarrow Y$ is a Baire-1 function, $X$ is completely metrisable and $Y$ is separable metrisable, then $f$ is continuous on a dense $G_{\delta}$ set (see [6, p. 193]). Since a multifunction $F: X \rightarrow P_{k}(Y)$ is continuous if and only if $F$ is continuous viewed as a single-valued function from $X$ into $\left(P_{k}(Y), \tau_{V}\right)$ (see for instance $[1$, p. 531]), we immediately have the following.

COROLlary 9. If $X$ is completely metrisable, $Y$ is separable metrisable and $F: X \rightarrow P_{k}(Y)$ is a Baire-1 multifunction, then $F$ is continuous on a dense $G_{\delta}$ set.

Combining the above corollary with Proposition 7, we get the following well-known fact from multivalued analysis (see [5, p. 73]). 
Corollary 10. If $X$ is completely metrisable, $Y$ is separable metrisable and $F: X \rightarrow P_{k}(Y)$ is a lower semicontinuous or upper semicontinuous multifunction, then $F$ is continuous on a dense $G_{\delta}$ set.

Besides the case of $P_{k}(Y)$-valued multifunctions, Baire-1 and weak Baire-1 multifunctions still behave well, at least with respect to lower semicontinuity, as the following proposition illustrates.

Proposit ION 11. If $X$ is completely metrisable, $Y$ is separable metrisable and $F: X \rightarrow 2^{Y} \backslash\{\emptyset\}$ is a weak Baire-1 multifunction, then $F$ is lower semicontinuous on a dense $G_{\delta}$ set.

Proof: Clearly $Y$ is second countable. Let $\left\{U_{n}\right\}_{n \geqslant 1}$ be a countable base for $Y$. Put $C_{n}=F^{-}\left(U_{n}\right)$. Since $F$ is a weak Baire-1 multifunction, $C_{n}$ is $F_{\sigma}$ for every $n \geqslant 1$. Put $D=\bigcup_{n \geqslant 1}\left(C_{n} \backslash \operatorname{int} C_{n}\right)$. Observe that since $C_{n} \in \Sigma_{2}^{0}(X)$ so is $C_{n} \backslash \operatorname{int} C_{n}$. Thus we have that $C_{n} \backslash$ int $C_{n}=\bigcup_{n \geqslant 1} F_{n}$, where $F_{n}$ are closed, nowhere dense subsets of $X$. So we conclude that $D^{c}$ is a dense $G_{\delta}$ set.

We claim that $F$ is lower semicontinuous on $D^{c}$. Indeed let $x \in D^{c}$ and $U \subseteq Y$ open be such that $F(x) \cap U \neq \emptyset$. Then there exists a $U_{n}$ basic open such that $F(x) \cap U_{n} \neq \emptyset$ which implies that $x \in C_{n}$. Since $x \in D^{c}$ we have that $x \in \operatorname{int} C_{n}$. Thus for any $y \in \operatorname{int} C_{n}$ we have that $F(y) \bigcap U \neq \emptyset$, which implies that $F$ is lower semicontinuous at $x$.

As before, combining Proposition 11 with Proposition 7 , we get the following result, due to Fort (see [4]).

Corollary 12. If $X$ is completely metrisable, $Y$ is separable metrisable and $F: X \rightarrow 2^{Y} \backslash\{\emptyset\}$ is an upper semicontinuous multifunction, then $F$ is continuous on a dense $G_{\delta}$ set.

We close this section by presenting two lemmas concerning weak Baire-1 multifunctions. As usual for any multifunction $F: X \rightarrow 2^{Y} \backslash\{\emptyset\}$, by $\bar{F}$ we denote the multifunction defined by $\bar{F}(x)=\overline{F(x)}$ for every $x \in X$.

LEMMA 13. A multifunction $F: X \rightarrow 2^{Y} \backslash\{\emptyset\}$ is a weak Baire-1 multifunction if and only if $\bar{F}$ is.

PROOF: Recall that for any $U \subseteq Y$ open, we have that $A \cap U \neq \emptyset$ if and only if $\bar{A} \cap U \neq \emptyset$.

LEMma 14. Let $F: X \rightarrow 2^{Y} \backslash\{\emptyset\}$ be a weak Baire-1 multifunction. Let $V \subseteq Y$ open and $C \subseteq X$ closed be such that $F(x) \cap V \neq \emptyset$ for every $x \in C$. Define $\widehat{F}: X \rightarrow$ $2^{Y} \backslash\{\emptyset\}$ by:

$$
\hat{F}(x)= \begin{cases}F(x) \cap V & : \quad x \in C \\ F(x) & : \quad x \notin C\end{cases}
$$

Then $\widehat{F}$ is a weak Baire-1 multifunction. 
Proof: Let $U \subseteq Y$ open and observe that $\widehat{F}^{-}(U)=F^{-}(U)$ if $U \cap V \neq \emptyset$ and that $\hat{F}^{-}(U)=F^{-}(U) \backslash C=F^{-}(U) \cap C^{c}$ otherwise. Since $C$ is closed, $C^{c}$ is open and in particular $F_{\sigma}$. Thus $\widehat{F}^{-}(U) \in \mathbf{\Sigma}_{2}^{0}(X)$ for any $U \subseteq Y$ open, as desired.

\section{Selections of Baire-1 Multifunctions}

It is well-known that an upper semicontinuous multifunction does not admit continuous selections. In fact if a multifunction $F: X \rightarrow 2^{Y} \backslash\{\emptyset\}$ is locally selectionable then $F$ is actually lower semicontinuous (recall that a multifunction $F$ is said to be locally selectionable if for every $[x, y] \in G r F$ there exists $U \in N(x)$ and $f$ a continuous selection of $\left.F\right|_{U}$ such that $f(x)=y$, see [5, p. 89]). So we have to abandon any hope for obtaining continuous selections for upper semicontinuous multifunctions.

However, as we show in Corollary 12, upper semicontinuous multifunctions actually have "many" points of continuity (and obviously of lower semicontinuity). It is natural then to expect that under some additional hypotheses, upper semicontinuous multifunctions have selections which are continuous on a dense $G_{\delta}$ set. Among all possible candidates, Baire-1 functions are the most prominent ones.

In this section we prove that this is the case. In fact we show much more. Specifically we prove that if $X$ is a metrisable space, $Y$ a Polish space and $F: X \rightarrow P_{f}(Y)$ a weak Baire-1 multifunction, then $F$ admits a Baire-1 selection. That is there exists a Baire1 function $f: X \rightarrow Y$ such that $f(x) \in F(x)$ for every $x \in X$. So according to Proposition 7, our selection theorem is applied to both lower semicontinuous and upper semicontinuous, closed-valued multifunctions.

It is worth mentioning that even if we are actually looking for generically continuous selections, our proof is modelled after the Kuratowski-Ryll Nardzewski selection theorem which provides measurable selections. As usual, we say that a function $f: X \rightarrow Y$ is $\Delta_{2}^{0}(X)$-measurable if and only if $f^{-1}(U) \in \Delta_{2}^{0}(X)$ for every $U \subseteq Y$ open, where $\Delta_{2}^{0}(X)$ is the ambiguous class (that is, $\Delta_{2}^{0}(X)=\Sigma_{2}^{0}(X) \cap \Pi_{2}^{0}(X)$ ).

Theorem 15. Let $X$ be a metrisable space, $Y$ a Polish space and $F: X \rightarrow P_{f}(Y)$ a weak Baire-1 multifunction. Then $F$ admits a Baire-1 selection.

PROOF: Let $d$ be a compatible metric for $Y$ with respect to which the $d$-diameter of $Y$ is strictly less than $1 / 2$. Fix $D=\left\{y_{i}\right\}_{i \geqslant 1}$ a countable dense subset of $Y$. Define $f_{1}: X \rightarrow Y$ by $f_{1}(x)=y_{1}$ for every $x \in X$, where $y_{1}$ is an arbitrary element of $D$. Clearly $f_{1}$ is continuous.

For every $n \geqslant 1$, we shall construct inductively a sequence $\left\{f_{n}\right\}_{n \geqslant 1}$ of $\Delta_{2}^{0}(X)$ measurable functions such that:

(i) $d\left(f_{n}(x), F(x)\right)<1 / 2^{n}$, for every $x \in X$;

(ii) $d\left(f_{n}(x), f_{n-1}(x)\right)<1 / 2^{n-1}$, for every $x \in X$.

Obviously $f_{1}$ is $\Delta_{2}^{0}(X)$-measurable and satisfies hypothesis (i). Suppose that $f_{1}$, 
$f_{2}, \ldots, f_{n-1}$ have been constructed. Now for every $i \geqslant 1$ define:

$$
A_{i}^{n}=\left\{x \in X: d\left(y_{i}, F(x)\right)<1 / 2^{n}\right\}=F^{-}\left(B\left(y_{i}, 1 / 2^{n}\right)\right)
$$

and

$$
C_{i}^{n}=\left\{x \in X: d\left(y_{i}, f_{n-1}(x)\right)<1 / 2^{n-1}\right\}=f_{n-1}^{-1}\left(B\left(y_{i}, 1 / 2^{n-1}\right)\right)
$$

where $y_{i} \in D$. Since $F$ is weak Baire-1 multifunction, we have that $A_{i}^{n} \in \Sigma_{2}^{0}(X)$ for every $i \geqslant 1$. On the other hand, since $f_{n-1}$ is $\Delta_{2}^{0}(X)$-measurable, we get that $C_{i}^{n} \in$ $\Delta_{2}^{0}(X) \subseteq \Sigma_{2}^{0}(X)$. Put $W_{i}^{n}=A_{i}^{n} \cap C_{i}^{n} \in \Sigma_{2}^{0}(X)$ for every $i \geqslant 1$. As in the proof of the Kuratowski-Ryll Nardzewski selection theorem (see for instance [5, p. 155]), using an argument based on the density of the set $D$, we can see that $X=\bigcup_{i \geqslant 1} W_{i}^{n}$. So by the generalised reduction property of $\boldsymbol{\Sigma}_{2}^{0}(X)$ sets, we can find a sequence $\left\{V_{i}^{n}\right\}_{i \geqslant 1}$ of $\Sigma_{2}^{0}(X)$ sets, such that $V_{i}^{n} \subseteq W_{i}^{n}, V_{i}^{n} \cap V_{j}^{n}=\emptyset$ if $i \neq j$ and $X=\bigcup_{i \geqslant 1} V_{i}^{n}$. Observe that $V_{i}^{n}=\left(\bigcup_{j \neq i} V_{j}^{n}\right)^{c}$ and so $V_{i}^{n} \in \Delta_{2}^{0}(X)$ for every $i \geqslant 1$.

Define $f_{n}: X \rightarrow Y$ by $f_{n}(x)=y_{i}$ if $x \in V_{i}^{n}$. It is clear that $f_{n}$ satisfies (i) and (ii). It remains to check that $f_{n}$ is $\Delta_{2}^{0}(X)$-measurable. Clearly it is enough to show that $f_{n}^{-1}(U) \in \Sigma_{2}^{0}(X)$ for any $U \subseteq Y$ open and that $f_{n}^{-1}(C) \in \Sigma_{2}^{0}(X)$ for any $C \subseteq Y$ closed. Let $U \subseteq Y$ open. Put $D_{U}=D \cap U$, which is at most countable (it might be empty of course). Then we have:

$$
f_{n}^{-1}(U)=\bigcup_{y_{i} \in D_{U}} f_{n}^{-1}\left(\left\{y_{i}\right\}\right)=\bigcup_{y_{i} \in D_{U}} V_{i}^{n} \in \Sigma_{2}^{0}(X) .
$$

Similarly if $C \subseteq Y$ is closed, put $D_{C}=D \cap C$ and observe that:

$$
f_{n}^{-1}(C)=\bigcup_{y_{i} \in D_{C}} f_{n}^{-1}\left(\left\{y_{i}\right\}\right)=\bigcup_{y_{i} \in D_{C}} V_{i}^{n} \in \Sigma_{2}^{0}(X) .
$$

So $f_{n}$ is $\Delta_{2}^{0}(X)$-measurable and the induction is complete.

Now define $f: X \rightarrow Y$ by $f(x)=\lim f_{n}(x)$ for every $x \in X$. From property (ii) we know that this limit exists ( $Y$ is complete), in fact uniformly in $x \in X$. Need to prove that $f$ is Baire-1 function. For this purpose it is enough to show that $f^{-1}(C) \in \Pi_{2}^{0}(X)$ for every $C \subseteq Y$ closed. So let $C \subseteq Y$ closed. From the fact that $f_{n} \rightarrow f$ uniformly, for every $k \geqslant 1$, there exists $n_{0}(k) \in \mathbb{N}$ such that $d\left(f_{n}(x), f(x)\right)<1 / k$ for every $n \geqslant n_{0}(k)$ and $x \in X$. Put $U_{k}=\{y \in Y: d(y, C)<1 / k\}$ for every $k \geqslant 1$. Clearly each $U_{k}$ is an open subset of $Y$. Using an argument which is rather folklore in measure theory (see for instance [1, p. 139, Lemma 4.28]) we can easily see that $f^{-1}(C)=\bigcap_{k \geqslant 1} f_{n_{0}(k)}^{-1}\left(U_{k}\right)$. Since each $f_{n}$ is $\Delta_{2}^{0}(X)$-measurable and $\Delta_{2}^{0}(X) \subseteq \Pi_{2}^{0}(X)$, we get that $f^{-1}(C) \in \Pi_{2}^{0}(X)$ as desired. So $f$ is indeed Baire-1. Finally from property (i), we have that $d(f(x), F(x))=0$ for every $x \in X$. Since $F$ is closed-valued, we conclude that $f(x) \in F(x)$ for all $x \in X$ and this completes the proof. 
REMARK 2. It is easy to see that Theorem 15 is still valid if $Y$ is separable metrisable and $F: X \rightarrow P_{k}(Y)$ a weak Baire-1 multifunction. Indeed let $d$ be a compatible metric for $Y$ with respect to which $(Y, d)$ is totally bounded. Denote by $\bar{Y}$ the completion of $Y$, which is compact metrisable and hence Polish. Observe that $F(x) \in P_{k}(Y) \subseteq P_{f}(\bar{Y})$ for every $x \in X$ and apply Theorem 15 .

The next proposition gives an equivalent characterisation of weak Baire-1, closedvalued multifunctions.

Proposition 16. Let $X$ be a metrisable space, $Y$ a Polish space and $F$ : $X \rightarrow P_{f}(Y)$ a multifunction. Then the following are equivalent.

(i) $F$ is a weak Baire-1 multifunction.

(ii) There exists a sequence $\left\{f_{n}\right\}_{n \geqslant 1}$ of Baire-1 selections of $F$ such that $F(x)={\overline{\left\{f_{n}(x)\right\}_{n \geqslant 1}}}_{n}$ for every $x \in X$.

Proof: (i) $\Rightarrow$ (ii) Let $D$ be as in Theorem 15. For every $i \geqslant 1$ and $n \geqslant 1$ put $C_{i n}=F^{-}\left(B\left(y_{i}, 2^{-n}\right)\right)$. Since $F$ is a weak Baire-1 multifunction $C_{i n} \in \Sigma_{2}^{0}(X)$. So it can be written as $C_{i n}=\bigcup_{k \geqslant 1} C_{\mathrm{ink}}$, where $C_{\text {ink }}$ are closed subsets of $X$ for every $i, n, k \geqslant 1$. Define $F_{\text {ink }}: X \rightarrow P_{f}(Y)$ by:

$$
F_{\text {ink }}(x)=\left\{\begin{array}{lll}
\overline{F(x) \cap B\left(y_{i}, 2^{-n}\right)} & : & x \in C_{\text {ink }} \\
F(x) & : & x \notin C_{\text {ink }}
\end{array}\right.
$$

From Lemma 13 and Lemma 14 we get that $F_{\text {ink }}$ is a weak Baire-1, closed-valued multifunction for every $i, n, k \geqslant 1$. Apply Theorem 15 and get a Baire-1 selection $f_{\text {ink }}$ of $F_{\text {ink }}$ for every $i, n, k \geqslant 1$. We claim that $\left\{f_{\text {ink }}\right\}_{i, n, k \geqslant 1}$ is the desired dense sequence. Indeed fix $x \in X$ and let $y \in F(x)$ and $\varepsilon>0$. Let $n \geqslant 1$ be such that $\varepsilon>2^{-n+1}$ and $y_{i} \in D$ such that $d\left(y, y_{i}\right)<2^{-n}$. Then since $x \in F^{-}\left(B\left(y_{i}, 2^{-n}\right)\right)$ we have that $x \in C_{\mathrm{ink}}$ for some $k \geqslant 1$. Let $f_{\text {ink }}$ be the selection of $F_{\text {ink }}$. Obviously $f_{\text {ink }}(x) \in \overline{B\left(y_{i}, 2^{-n}\right)}$. So we have that:

$$
d\left(y, f_{\text {ink }}(x)\right)<d\left(y, y_{i}\right)+d\left(y_{i}, f_{\text {ink }}(x)\right) \leqslant \frac{1}{2^{n}}+\frac{1}{2^{n}}=\frac{1}{2^{n-1}}<\varepsilon
$$

and we are done.

(ii) $\Rightarrow$ (i) Observe that for any $U \subseteq Y$ open, we have that:

$$
\begin{aligned}
F^{-}(U) & =\{x \in X: F(x) \cap U \neq \emptyset\}=\bigcup_{n \geqslant 1}\left\{x \in X: f_{n}(x) \in U\right\} \\
& =\bigcup_{n \geqslant 1} f_{n}^{-1}(U) \in \Sigma_{2}^{0}(X) .
\end{aligned}
$$

So $F$ is a weak Baire-1 multifunction and the proof is completed.

Remark 3. We can easily see (as in Remark 2) that Proposition 16 is still valid if $Y$ is a separable metrisable space and $F: X \rightarrow P_{k}(Y)$ a weak Baire-1 multifunction. 
Recall the following fundamental fact, due to Kuratowski. Its proof can be found in [6, p. 173].

THEOREM 17. Let $(X, \tau)$ be a Polish space and $\left\{A_{n}\right\}_{n \geqslant 1}$ a sequence of $\Delta_{\xi}^{0}(X)$ sets. Then there exists a Polish topology $\tau^{\prime}$ on $X$ such that $\tau^{\prime} \supseteq \tau, \tau^{\prime} \subseteq \Sigma_{\xi}^{0}(X, \tau)$ and $A_{n}$ is clopen in $\left(X, \tau^{\prime}\right)$ for every $n \geqslant 1$.

Now assume that $X$ is also Polish. In this case, using Theorem 17, we can have an improved version of Proposition 16.

Proposition 18. Let $(X, \tau)$ be a Polish space. Let also $Y$ be a Polish space and $F: X \rightarrow P_{f}(Y)$ a multifunction. Then the following are equivalent.

(i) $F$ is a weak Baire-1 multifunction.

(ii) There exist a Polish topology $\tau^{\prime}$ on $X$, such that $\tau^{\prime} \subseteq \Sigma_{2}^{0}(X, \tau)$, and a sequence $\left\{f_{n}\right\}_{n \geqslant 1}$ of $\tau^{\prime}$-continuous selections of $F$ such that $F(x)=$

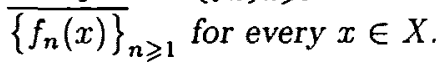

Proof: (i) $\Rightarrow$ (ii) Apply Proposition 16 and get a sequence $\left\{f_{n}\right\}_{n \geqslant 1}$ of Baire-1 selections of $F$ such that $F(x)={\overline{\left\{f_{n}(x)\right\}_{n \geqslant 1}}}_{n}$ for every $x \in X$. Let $\left\{V_{i}\right\}_{i \geqslant 1}$ be a countable base for $Y$. For every $n, i \geqslant 1$, let $A_{n i}=f_{n}^{-1}\left(V_{i}\right) \in \Sigma_{2}^{0}(X)$. By definition, each $A_{n i}$ can be written as $A_{n i}=\bigcup_{m \geqslant 1} C_{\text {nim }}$, where $C_{\text {nim }} \in \mathbf{\Pi}_{1}^{0}(X) \subseteq \Delta_{2}^{0}(X)$ for every $n, i, m \geqslant 1$. Apply Theorem 17 to the sequence $\left\{C_{\text {nim }}\right\}_{n, i, m \geqslant 1} \subseteq \Delta_{2}^{0}(X)$ and get a Polish topology $\tau^{\prime}$ on $X$, with $\tau^{\prime} \subseteq \Sigma_{2}^{0}(X, \tau)$, such that $C_{\text {nim }}$ is $\tau^{\prime}$-clopen for every $n, i, m \geqslant 1$. Observe that $A_{n i} \in \tau^{\prime}$. So each $f_{n}$ is $\tau^{\prime}$-continuous, and we are done.

(ii) $\Rightarrow$ (i) Since $\tau^{\prime} \subseteq \Sigma_{2}^{0}(X, \tau)$ and each $f_{n}$ is $\tau^{\prime}$-continuous, we get that $f_{n}^{-1}(U) \in \Sigma_{2}^{0}(X, \tau)$ for any $U \subseteq Y$ open. Thus each $f_{n}$ is a Baire-1 function for the $\tau$ topology of $X$. So from Proposition 16, we conclude that $F$ is a weak Baire-1 multifunction.

In contrast to the remarks at the beginning of this section, combining Proposition 18 with Proposition 7 , we get the following interesting corollary.

Corollary 19. Let $(X, \tau)$ be a Polish space. Let also $Y$ be a Polish space and $F: X \rightarrow P_{f}(Y)$ an upper semicontinuous or lower semicontinuous multifunction. Then there exist a Polish topology $\tau^{\prime} \subseteq \Sigma_{2}^{0}(X, \tau)$ on $X$ and a sequence $\left\{f_{n}\right\}_{n \geqslant 1}$ of $\tau^{\prime}$-continuous selections of $F$ such that $F(x)={\overline{\left\{f_{n}(x)\right\}}}_{n \geqslant 1}$ for every $x \in X$.

\section{Weak* Usco and Cusco Multifunctions}

Let $X$ be an infinite dimensional Banach space. By $X^{*}$ we denote the topological dual of $X$. The norms of $X$ and $X^{*}$ and the duality brackets for the pair $\left(X, X^{*}\right)$ will be denoted by $\|\cdot\|,\|\cdot\|_{X} \cdot$ and $\langle\cdot, \cdot\rangle$ respectively. Finally by $P_{f c}(X)$ we denote the collection of all nonempty, closed, convex subsets of $X$, while by $P_{w k}\left(X_{w *}^{*}\right)$ (respectively by $\left.P_{w k c}\left(X_{w *}^{*}\right)\right)$ the collection of all nonempty, weakly* compact (respectively and convex) subsets of $X^{*}$. 
Let $Z$ be a completely metrisable space. An upper semicontinuous multifunction $F: Z \rightarrow P_{w k}\left(X_{w^{*}}^{*}\right)$ (on $X^{*}$ we consider the weak* topology) is called a weak* $u s c o$. If in addition $F(x)$ is convex (that is if $F(x) \in P_{w k c}\left(X_{w *}^{*}\right)$ ) for any $x \in X$, then $F$ is said to be a weak ${ }^{*}$ cusco. Note that in the general definition of a weak* usco, $Z$ is only assumed to be a Hausdorff topological space. We make the complete metrisability assumption on $Z$ in order to apply our previous results. However we should point out that this does not reduce the range of applications. Many important multifunctions (such as maximal monotone operators, subdifferentials of locally Lipschitz functionals) are weak* cuscos.

In the following theorem we shall show that if $X^{*}$ is separable, weak* uscos possess strong continuity properties. Our proof is essentially based on the techniques developed in the previous two sections.

THEOREM 20. Let $Z$ be a completely metrisable space, $X$ be a Banach space such that $X^{*}$ is separable and $F: Z \rightarrow P_{w k}\left(X_{w^{*}}^{*}\right)$ be a weak* usco. Then $F$ is norm lower semicontinuous on a dense $G_{\delta}$ set.

Proof: Observe that our hypothesis on $X$ entails that $X^{*}$ equipped with the strong topology (still denoted by $X_{s}^{*}$ ) has the structure of a Polish space. Note that $F$ can be viewed as a multifunction from $Z$ into $P_{f}\left(X_{s}^{*}\right)$. We claim that $F$, viewed this way, is a weak Baire-1 multifunction. Indeed let $U \subseteq X^{*}$ be strongly open. Then $U$ can be written as $U=\bigcup_{n \geqslant 1} \overline{B_{n}}$, where $\overline{B_{n}}$ are closed balls in $X^{*}$. We have that:

$$
F^{-}(U)=F^{-}\left(\bigcup_{n \geqslant 1} \overline{B_{n}}\right)=\bigcup_{n \geqslant 1} F^{-}\left(\overline{B_{n}}\right) .
$$

Since $F$ is a weak* usco and each $\overline{B_{n}}$ is weakly* closed, we get that $F^{-}\left(\overline{B_{n}}\right)$ is closed in $Z$ for every $n \geqslant 1$. So we conclude that $F^{-}(U) \in \Sigma_{2}^{0}(Z)$, which implies that $F$ is indeed a weak Baire-1 multifunction. Then from Proposition 11 we get that $F$ is norm lower semicontinuous on a dense $G_{\delta}$ subset of $Z$.

REMARK 4. We shall give an alternative proof of Theorem 20 based on Proposition 16. Indeed since $F$ is a weak Baire-1, closed-valued multifunction, pick a sequence $\left\{f_{n}\right\}_{n \geqslant 1}$ of Baire-1 selections of $F$ such that $F(x)={\overline{\left\{f_{n}(x)\right\}_{n}}}_{n \geq 1}$ for every $x \in X$. Denote by $C_{n}$ the set of points of continuity of each $f_{n}$. Since $Z$ is completely metrisable and $f_{n}$ is Baire-1, $C_{n}$ is a dense $G_{\delta}$ set for every $n \geqslant 1$. Put $C=\bigcap_{n \geqslant 1} C_{n}$, which is dense $G_{\delta}$. We claim that $F$ is norm lower semicontinuous on $C$. Indeed let $z \in C$ and $U \subseteq X^{*}$ strongly open, such that $F(z) \cap U \neq \emptyset$. Then there exists $n \geqslant 1$ such that $f_{n}(z) \subseteq U$. From the norm continuity of $f_{n}$ at $z$, we may find $V \in N(z)$ such that $f_{n}(V) \subseteq U$. But then observe that $F^{-}(U) \supseteq V$, which implies that $F$ is norm lower semicontinuous at $z$.

Recall that for any function $f: X \rightarrow Y$ and any set $A \subseteq Y$ we have that $f^{-1}(A)$ $=f^{-}(A)=f^{+}(A)$. So an immediate corollary of Theorem 20 is the following. 
Corollary 21. Let $Z$ be a completely metrisable space, $X$ be a Banach space such that $X^{*}$ is separable and $f: Z \rightarrow X^{*}$ a weakly* continuous function. Then $f$ is norm continuous on a dense $G_{\delta}$ set.

Now assume that $F$ has strongly compact values. In this case $F$ possesses even stronger continuity properties.

Proposition 22. Let $Z$ be a completely metrisable space, $X$ be a Banach space such that $X^{*}$ is separable and $F: Z \rightarrow P_{k}\left(X^{*}\right)$ a weak* usco. Then $F$ is norm continuous on a dense $G_{\delta}$ set.

Proof: We shall show that in this case $F$ is a Baire-1 multifunction. We already know from the proof of Theorem 20 that $F$ is a weak Baire-1 multifunction. So it is enough to show that $F^{+}(U) \in \Sigma_{2}^{0}(Z)$ for every $U \subseteq X^{*}$ strongly open. Let $U \subseteq X^{*}$ be strongly open. Write $U$ as $U=\bigcup_{n \geqslant 1} B_{n}=\bigcup_{n \geqslant 1} \overline{B_{n}}$, where each $B_{n}$ (respectively $\overline{B_{n}}$ ) is an open (respectively closed) ball in $X^{*}$. We have that:

$$
F^{+}(U)=F^{+}\left(\bigcup_{n \geqslant 1} B_{n}\right)=F^{+}\left(\bigcup_{n \geqslant 1} \overline{B_{n}}\right) \text {. }
$$

Let $\left\{V_{i}\right\}_{i \geqslant 1}$ be the (countable) collection of all finite unions of $\overline{B_{n}}$ 's. Then each $V_{i}$ is weakly* closed and:

$$
F^{+}(U)=F^{+}\left(\bigcup_{n \geqslant 1} B_{n}\right)=F^{+}\left(\bigcup_{n \geqslant 1} \overline{B_{n}}\right)=F^{+}\left(\bigcup_{i \geqslant 1} V_{i}\right) .
$$

We claim that:

$$
F^{+}\left(\bigcup_{n \geqslant 1} B_{n}\right)=\bigcup_{i \geqslant 1} F^{+}\left(V_{i}\right)
$$

It is clear that $F^{+}\left(\bigcup_{n \geqslant 1} B_{n}\right) \supseteq \bigcup_{i \geqslant 1} F^{+}\left(V_{i}\right)$. So let $x \in F^{+}\left(\bigcup_{n \geqslant 1} B_{n}\right)$. Then the sequence $\left\{B_{n}\right\}_{n \geqslant 1}$ forms an open cover of $F(x)$. From the strong compactness of $F(x)$, we may find $\left\{B_{n}\right\}_{n=1}^{k}$ such that $F(x) \subseteq \bigcup_{n=1}^{k} B_{n}$. From the definition of $V_{i}$ 's, there exists some $i \geqslant 1$ such that $V_{i}=\bigcup_{n=i}^{k} \overline{B_{n}}$. So $x \in F^{+}\left(V_{i}\right)$ and the claim is proved. Since each $V_{i}$ is weakly* closed and $F$ is weak* usco, we have that $F^{+}\left(V_{i}\right)$ is closed for every $i \geqslant 1$. Thus we conclude that $F^{+}(U) \in \Sigma_{2}^{0}(Z)$ for every $U \subseteq X^{*}$ strongly open, which implies that $F$ is indeed a Baire-1 multifunction. From Corollary 9 we get that $F$ is norm continuous on a dense $G_{\delta}$ set.

\section{A Final REMARK}

It is clear that the generalised reduction property of $\Sigma_{2}^{0}(X)$ sets is the essential ingredient of Theorem 15 (as well as of Proposition 18). In this section, we shall show that Theorem 15 is actually equivalent to the generalised reduction property of $\Sigma_{2}^{0}(X)$ sets. Let us be precise. 
Proposition 23. Let $X$ be a metrisable space. Then the following are equivalent.

(i) The class $\Sigma_{2}^{0}(X)$ has the generalised reduction property.

(ii) For any Polish space $Y$ and any weak Baire-1 multifunction $F: X \rightarrow$ $P_{f}(Y)$, there exists a Baire-1 function $f: X \rightarrow Y$, such that $f(x) \in F(x)$ for every $x \in X$.

Proof: Theorem 15 shows (i) $\Rightarrow$ (ii). To prove (ii) $\Rightarrow$ (i) let $\left\{A_{n}\right\}_{n \geqslant 1}$ be sequence of $\Sigma_{2}^{0}(X)$ subsets of $X$. We shall prove first the case when $X=\bigcup_{n \geqslant 1} A_{n}$. Denote by $Y$ the space $\mathbb{N}_{+}$of all the nonzero integers, equipped with the discrete topology. Then $Y$ is Polish. A compatible metric is $\delta(n, m)=0$ if $n \neq m$ and $\delta(n, m)=1$ if $n=m$ (which is also an ultrametric). Define $F: X \rightarrow 2^{Y} \backslash\{\emptyset\}$ by $F(x)=\left\{n: x \in A_{n}\right\}$ for every $x \in X$. Obviously $F$ is closed-valued. We claim that $F$ is weak Baire-1 multifunction. Indeed let $I \subseteq \mathbb{N}_{+}$and observe that:

$$
\begin{aligned}
F^{-}(I) & =\left\{x \in X: x \in A_{i}, i \in I\right\}=\bigcup_{i \in I} F^{-}(\{i\}) \\
& =\bigcup_{i \in I} A_{i} \in \Sigma_{2}^{0}(X) .
\end{aligned}
$$

So, from hypothesis, there exists a Baire-1 selection $f$ of $F$. Define $B_{n}=f^{-1}(\{n\})$ for every $n \geqslant 1$. Then $B_{n} \in \Sigma_{2}^{0}(X)$ (since $\{\dot{n}\}$ is open in $Y$ ), $B_{n} \subseteq A_{n}, B_{n} \cap B_{m}=\emptyset$ if $n \neq m$ and $X=\bigcup_{n \geqslant 1} B_{n}$.

For the general case put $Z=\bigcup_{n \geqslant 1} A_{n}$. Then $Z \in \Sigma_{2}^{0}(X)$. Note that $A_{n} \in \Sigma_{2}^{0}(Z)$ for every $n \geqslant 1$. Indeed, write $A_{n}=\bigcup_{m \geqslant 1} C_{m}$, where $C_{m}$ is closed in $X$ for every $m \geqslant 1$. Then we have that $A_{n}=\bigcup_{m \geqslant 1}\left(Z \cap C_{m}\right)$, where $Z \cap C_{m}$ is closed in $Z$ (the latter equipped with the relative topology) for every $m \geqslant 1$. Obviously $Z$ is metrisable. Define $F: Z \rightarrow P_{f}(Y)$ as before and obtain a sequence $\left\{B_{n}\right\}_{n \geqslant 1}$ of $\Sigma_{2}^{0}(Z)$ subsets of $Z$ such that $B_{n} \subseteq A_{n}$, $B_{n} \cap B_{m}=\emptyset$ if $n \neq m$ and $Z=\bigcup_{n \geqslant 1} B_{n}$. But then observe that $B_{n} \in \Sigma_{2}^{0}(X)$ for every $n \geqslant 1$. So we conclude that the class $\Sigma_{2}^{0}(X)$ has the generalised reduction property, as desired.

Remark 5. One should compare Proposition 23 with the following result due to Michael (see [10]). Let $X$ be a topological space. If for any Banach space $Y$, every lower semicontinuous multifunction $F: X \rightarrow P_{f c}(Y)$ admits a continuous selection, then $X$ is paracompact.

\section{REFERENCES}

[1] C.D. Aliprantis and K.C. Border, Infinite dimensional analysis, (Second edition) (Springer-Verlag, Berlin, 1999). 
[2] F. Deutsch, 'A survey of continuous selections', Contemp. Math. 18 (1983), 49-71.

[3] R. Engelking, General topology, Sigma Series in Pure Mathematics 6, (Second edition) (Heldermann-Verlag, Berlin, 1989).

[4] M.K. Fort, 'Category theorems', Fund. Math. 42 (1955), 276-288.

[5] S. Hu and N.S. Papageorgiou, Handbook of multivalued analysis, Volume I: Theory, Mathematics and its Applications 419 (Kluwer, Dordrecht, 1997).

[6] A.S. Kechris, Classical descriptive set theory, Graduate Texts in Math. 156 (Springer-Verlag, New York, 1995).

[7] P.S. Kenderov, 'Semicontinuity of set valued monotone mappings', Fund. Math. 88 (1975), 61-69.

[8] K. Kuratowski, Topology, I (Academic Press, New York, London, 1966).

[9] K. Kuratowski and C. Ryll-Nardzewski, 'A general theorem on selectors', Bull. Acad. Polon. Sci. Sér. Sci. Math. Astronom. Phys. 13 (1965), 397-403.

[10] E. Michael, 'Continuous selections I', Ann. Math. 63 (1956), 361-382.

[11] E. Odell and H.P. Rosenthal, 'A double dual characterization of separable Banach spaces containing $l_{1}$ ', Istael J. Math. 20 (1975), 375-384.

Department of Mathematics

Faculty of Applied Sciences

National Technical University of Athens

Athens

Greece 